\title{
Explicit Analytic Solution for the Nonlinear Evolution Equations using the Simplest Equation Method
}

\author{
Mohammed. K. Elboree* \\ Mathematics Department, Faculty of Science, South Valley University, Qena, Egypt
}

Received: 15 Jun. 2013, Revised: 11 Oct. 2013, Accepted: 12 Oct. 2013

Published online: 1 Jan. 2014

\begin{abstract}
In this paper, we focus on the exact solution of the coupled nonlinear Schrödinger type equation by means of the simplest equation method for the cases when the simplest equation is the equation of Bernoulli or the equation of Riccati. We use the appropriate ansätz to convert the above-mentioned equations to an ordinary differential equation and reduced to nonlinear algebraic systems of relationships among the parameters of the equations and the parameters of the solution. The method used here can be also extended to many other nonlinear partial differential equations. The properties of some solutions for this system are shown by some figures.
\end{abstract}

Keywords: Exact solutions; coupled nonlinear Schrödinger type equation; Bernoulli equation; Riccati equation

\section{Introduction}

As is well-known, many phenomena in physics, mechanics, chemistry, biology, etc. are often described by nonlinear partial differential equations (PDEs), such as Korteweg Vries equation, Burgers equation, Schrödinger equation, Boussinesq equation and so on. When we want to understand the physical mechanism of phenomena in nature, described by nonlinear PDEs, exact solutions have to be explored. These solutions has long been a major concern for both mathematicians and physicists. So we search for a mathematical algorithm to discover the exact solutions of nonlinear partial differential equations. Many papers has been focused on the application of the known methods to construct the solutions of nonlinear evolution equation (NLEE), among them the inverse scattering transform [[1]-[3]] and Hirota method [4], tanh method $[1,2,3,4,5,6]$, multiple exp-function method [7], Backlund transformation method [8], simplest equation method $[9,10]$, and so on.

From the standpoint of application, nonlinear partial differential equations describe the motion of the isolated waves, such as in physics, in which applications extend over water surface, gravity waves, and electromagnetic radiation reactions, chemistry, and several other fields.

The simplest equation method is a very powerful mathematical technique for finding exact solutions of nonlinear ordinary differential equations. It has been developed by Kudryashov $[9,10]$ and used successfully by many authors for finding exact solutions of ODEs in mathematical physics [11].

This paper is organized as follows. In section 2, we give a brief algorithm for the simplest equation method. In section 3, we apply this method to the coupled nonlinear Schrödinger type equation and find out several exact solutions for this model. We give the conclusion in the section 4 .

\section{The algorithm of the simplest equation method}

For given a nonlinear equation

$$
H\left(u, u_{t}, u_{x}, u_{x t}, \ldots\right)=0 .
$$

The main steps of our proposed are given as follows Step 1. By using the wave transformation $u(x, t)=u(\zeta), \zeta=$ $k_{1} x+\omega_{1} t+\xi_{0}, \varphi_{0}, \xi_{0}$ are constants, we can convert Eq. (1) into an ordinary differential equation (ODE)

$$
H\left(u, u_{\zeta}, u_{\zeta \zeta}, \ldots\right)=0 \text {. }
$$
forms

Step 2. We seek the solution of Eq.(2) in the following

$$
u(\zeta)=\sum_{i=0}^{n} a_{i} f^{i}(\zeta)
$$

\footnotetext{
*Corresponding author e-mail: mm_kalf@yahoo.com
} 
which $a_{i}(i=0,1,2, \ldots, n)$ are constants to be determined. The balancing number $n$ is a positive integer which can be determined by balancing the highest order derivative terms with the highest power nonlinear terms in Eq.(2) and $f(\zeta)$ are the functions that satisfy the simplest equations (ordinary differential equations). The simplest equation has two properties, first it is lesser order than Eq.(2) and we know the general solution of the simplest equation [12].

In this paper, we shall use as simplest equation the equations of Bernoulli and Riccati which are well known nonlinear ordinary differential equations and their solutions can be expressed by elementary functions. For the Bernoulli equation

$$
f^{\prime}(\zeta)=a f(\zeta)+b f^{2}(\zeta)
$$

We found that the use of the Bernoulli equation leads to new traveling-wave and wavefront solutions of Eq.(1). Eq.(4) admits the following exact solutions

$$
f(\zeta)=\frac{a \exp \left[a\left(\zeta+\xi_{0}\right)\right]}{1-b \exp \left[a\left(\zeta+\xi_{0}\right)\right]}
$$

when $a>0, b<0$ and

$$
f(\zeta)=\frac{a \exp \left[a\left(\zeta+\xi_{0}\right)\right]}{1+b \exp \left[a\left(\zeta+\xi_{0}\right)\right]}
$$

when $a<0, b>0$ and $\xi_{0}$ is a constant of integration. For the Riccati equation

$$
f^{\prime}(\zeta)=\alpha f^{2}(\zeta)+\beta
$$

Eq.(7) admits the following exact solutions [13]

$f(\zeta)=-\frac{\sqrt{-\alpha \beta}}{\alpha} \tanh \left(\sqrt{-\alpha \beta} \zeta-\frac{v \ln \left(\xi_{0}\right)}{2}\right), \quad \xi_{0}>0, \quad v= \pm 1$,

where $\alpha \beta<0$, and

$$
f(\zeta)=\frac{\sqrt{\alpha \beta}}{\alpha} \tan \left(\sqrt{\alpha \beta} \zeta+\xi_{0}\right), \quad \xi_{0}=\text { constant },
$$

where $\alpha \beta>0$.

Step 3. Derive the algebraic system for the coefficients $a_{i}(i=0,1,2, \ldots, n)$ to generate the system for the unknown coefficients $a_{i}(i=0,1,2, \ldots, n), a, b$ and wave parameter $c$, substituting Eq.(3) into Eq.(2) with Eq.(4) and set the coefficients of the polynomial to zero yields a set of algebraic equations for $a_{i}, a, b, c$.

Step 4. Solving the nonlinear parameterized algebraic system and substituting the results into Eq.(3) to obtain the exact traveling wave solutions for Eq.(1).

Remark. When $a=\delta, b=-1$ the Eq.(4) has a another form of Bernoulli equation

$$
f^{\prime}(\zeta)=\delta f(\zeta)-f^{2}(\zeta)
$$

which has the exact solutions when $\delta>0$,

$$
f(\zeta)=\frac{\delta}{2}\left[1+\tanh \left(\frac{\delta}{2}\left(\zeta+\xi_{0}\right)\right)\right],
$$

and when $\delta<0$

$$
f(\zeta)=\frac{\delta}{2}\left[1-\tanh \left(\frac{\delta}{2}\left(\zeta+\xi_{0}\right)\right)\right] .
$$

\section{The simplest equation method for the coupled nonlinear Schrödinger type equation}

Now we consider the new coupled nonlinear Schrödinger type (CNLST) equation

$$
\begin{aligned}
& u_{x t}=u_{x x}+\frac{2}{1-\beta^{2}}|u|^{2} u+u(v-w), \\
& v_{t}=-\frac{\left(|u|^{2}\right)_{t}}{1+\beta}+(1+\beta) v_{x} \\
& w_{t}=\frac{\left(|u|^{2}\right)_{t}}{1-\beta}+(1-\beta) w_{x} .
\end{aligned}
$$

Where $\beta$ is a real constant, $|\beta| \neq 1$, was proposed by Ma and Geng via a spectral problem and its auxiliary one [14]. By using the transformation

$u(x, t)=U(\zeta) e^{i\left(k_{2} x-\omega_{2} t+\varphi_{0}\right)}, \quad v(x, t)=V(\zeta), \quad w(x, t)=W(\zeta)$,

where $\zeta=k_{1} x+\omega_{1} t+\xi_{0}, \varphi_{0}, \xi_{0}$ are constants.

Substituting Eq.(14) into Eq.(13), we obtain

$$
\begin{aligned}
& \left(k_{1}^{2}+k_{1} \omega_{1}\right) U^{\prime \prime}-\left(k_{2}^{2}+k_{2} \omega_{2}\right) U+\frac{2}{1-\beta^{2}} U^{3}+U(V-W)=0 \\
& \omega_{1} V^{\prime}+\frac{2 \omega_{1}}{1+\beta} U U^{\prime}+(1+\beta) k_{1} V^{\prime}=0 \\
& \omega_{1} W^{\prime}-\frac{2 \omega_{1}}{1-\beta} U U^{\prime}+(1-\beta) k_{1} W^{\prime}=0
\end{aligned}
$$

Where the prime denotes to the derivative with respect to the variable $\zeta$. From the imaginary part of Eq.(13) we find $\omega_{2}=-\frac{k_{2}}{k_{1}}\left(\omega_{1}+2 k_{1}\right)$. Integrating Eq.(15), we obtain the relation between the variables $V(\zeta), U(\zeta)$ and $W(\zeta)$, $U(\zeta)$ as follows

$$
\begin{aligned}
& V(\zeta)=-\frac{\omega_{1}}{(1+\beta)\left(\omega_{1}+k_{1}(1+\beta)\right)} U(\zeta)^{2}, \\
& W(\zeta)=\frac{\omega_{1}}{(1-\beta)\left(\omega_{1}+k_{1}(1-\beta)\right)} U(\zeta)^{2} .
\end{aligned}
$$

Substituting $V(\zeta), W(\zeta)$ in Eq.(8), we obtain

$$
U^{\prime \prime}-\left(\frac{k_{2}}{k_{1}}\right)^{2} U-\frac{1-\beta^{2}}{\left(\omega_{1}+k_{1}\right)^{2}-k_{1}^{2} \beta^{2}} U^{3}=0 .
$$

Exact solution for Eq.(15) can be constructed as a finite series

$$
U(\zeta)=\sum_{i=0}^{N} c_{i} f^{i}(\zeta)
$$

where $f(\zeta)$ is a solution of some ordinary differential equation referred to as the simplest equation.

Balancing $U^{\prime \prime}$ with $U^{3}$, we find that $N=1$.

Substituting Eq.(18) along with Eq.(4) in Eq.(17) and then setting the coefficients of $f^{i}(i=1,2,3,4)$ to zero in the resultant expression, we obtain a set of algebraic 
equations for $\omega_{1}, k_{1}, k_{2}, a, b, c_{0}, c_{1}, \alpha, \beta$ as follows

$$
\begin{aligned}
& \frac{\left(1-\beta^{2}\right) c_{0}^{3}}{\left(k_{1}+\omega_{1}\right)^{2}-\beta^{2} k_{1}^{2}}+\frac{c_{0} k_{2}^{2}}{k_{1}^{2}}=0 \\
& c_{1}\left(a^{2}+\frac{3\left(1-\beta^{2}\right) c_{0}^{2}}{\left(k_{1}+\omega_{1}\right)^{2}-\beta^{2} k_{1}^{2}}+\frac{k_{2}^{2}}{k_{1}^{2}}\right)=0 \\
& 3 c_{1}\left(a b+\frac{\left(1-\beta^{2}\right) c_{0} c_{1}}{\left(k_{1}+\omega_{1}\right)^{2}-\beta^{2} k_{1}^{2}}\right)=0 \\
& 2 b^{2} c_{1}+\frac{\left(1-\beta^{2}\right) c_{1}^{3}}{\left(k_{1}+\omega_{1}\right)^{2}-\beta^{2} k_{1}^{2}}=0 .
\end{aligned}
$$

With the aid of Mathematica, we find the special solutions of the above system as follows

\section{Case 1:}

$c_{0}= \pm \frac{a \sqrt{\left(1-\beta^{2}\right) k_{1}^{2}+2 k_{1} \omega_{1}+\omega_{1}^{2}}}{\sqrt{2\left(\beta^{2}-1\right)}}$,

$c_{1}= \pm \frac{b \sqrt{2\left(\left(1-\beta^{2}\right) k_{1}^{2}+2 k_{1} \omega_{1}+\omega_{1}^{2}\right)}}{\sqrt{\beta^{2}-1}}$

$k_{2}= \pm \frac{a k_{1}}{\sqrt{2}}$

Using solution (5) of Eq.(4). Then the exact solution to Eq.(13) can be written as

$$
\begin{aligned}
u(x, t)= & \pm \frac{\sqrt{\left(1-\beta^{2}\right) k_{1}^{2}+2 k_{1} \omega_{1}+\omega_{1}^{2}}}{\left.\sqrt{\left(\beta^{2}-1\right.}\right)} e^{i\left( \pm \frac{a}{\sqrt{2}}\left(k_{1} x+\left(\omega_{1}+2 k_{1}\right) t\right)+\phi_{0}\right)} \\
& \left(\frac{a b \sqrt{2} \exp \left(a\left(\omega_{1} t+k_{1} x+\xi_{0}\right)\right)}{1-b \exp \left(a\left(\omega_{1} t+k_{1} x+\xi_{0}\right)\right)}+\frac{a}{\sqrt{2}}\right) \\
v(x, t)=\mp & \frac{\omega_{1}}{(1+\beta)\left(\omega_{1}+k_{1}(1+\beta)\right)}\left(\frac{\sqrt{\left(1-\beta^{2}\right) k_{1}^{2}+2 k_{1} \omega_{1}+\omega_{1}^{2}}}{\left.\sqrt{\left(\beta^{2}-1\right)}\right)^{2}}\right. \\
& \left(\left(\frac{a b \sqrt{2} \exp \left(a\left(\omega_{1} t+k_{1} x+\xi_{0}\right)\right)}{1-b \exp \left(a\left(\omega_{1} t+k_{1} x+\xi_{0}\right)\right)}+\frac{a}{\sqrt{2}}\right)\right)^{2}, \\
w(x, t)= & \mp \frac{\omega_{1}}{(1+\beta)\left(\omega_{1}+k_{1}(1+\beta)\right)}\left(\frac{\sqrt{\left(1-\beta^{2}\right) k_{1}^{2}+2 k_{1} \omega_{1}+\omega_{1}^{2}}}{\left.\sqrt{\left(\beta^{2}-1\right.}\right)}\right)^{2} \\
& \left(\left(\frac{a b \sqrt{2} \exp \left(a\left(\omega_{1} t+k_{1} x+\xi_{0}\right)\right)}{1-b \exp \left(a\left(\omega_{1} t+k_{1} x+\xi_{0}\right)\right)}+\frac{a}{\sqrt{2}}\right)\right)^{2},
\end{aligned}
$$

when $a>0, b<0$. Similarly for $a<0, b>0$.

\section{Case 2:}

$$
\begin{aligned}
& c_{0}= \pm \frac{a \sqrt{\left(1-\beta^{2}\right) k_{1}^{2}+2 k_{1} \omega_{1}+\omega_{1}^{2}}}{\sqrt{2\left(\beta^{2}-1\right)}} \\
& c_{1}= \pm \frac{b \sqrt{2\left(\left(1-\beta^{2}\right) k_{1}^{2}+2 k_{1} \omega_{1}+\omega_{1}^{2}\right)}}{\sqrt{\beta^{2}-1}}, \\
& k_{2}=\mp \frac{a k_{1}}{\sqrt{2}} .
\end{aligned}
$$
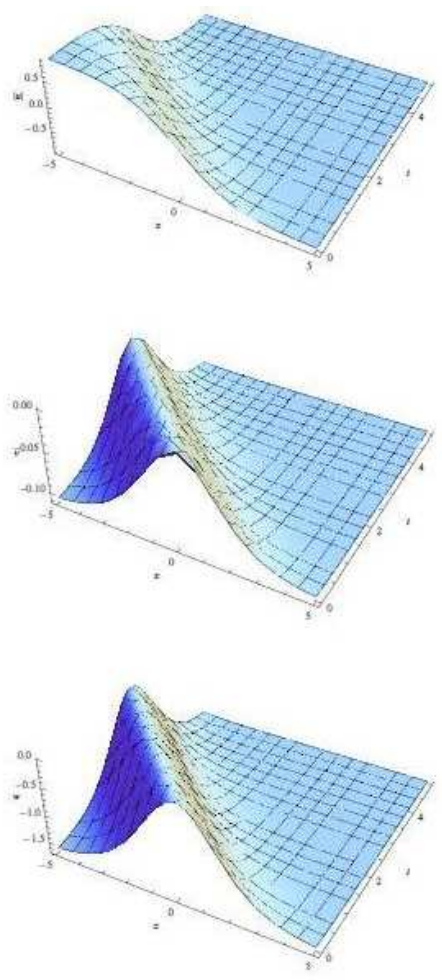

Fig. 1: The solutions of Eq.(13) when $a>0, b<0$.

Using solution (5) of Eq.(4). Then the exact solution to Eq.(13) can be written as

$$
\begin{aligned}
u(x, t)= & \pm \frac{\sqrt{\left(1-\beta^{2}\right) k_{1}^{2}+2 k_{1} \omega_{1}+\omega_{1}^{2}}}{\left.\sqrt{\left(\beta^{2}-1\right.}\right)} e^{i\left(\mp \frac{a}{\sqrt{2}}\left(k_{1} x+\left(\omega_{1}+2 k_{1}\right) t\right)+\phi_{0}\right)} \\
& \left(\frac{a b \sqrt{2} \exp \left(a\left(\omega_{1} t+k_{1} x+\xi_{0}\right)\right)}{1-b \exp \left(a\left(\omega_{1} t+k_{1} x+\xi_{0}\right)\right)}+\frac{a}{\sqrt{2}}\right)
\end{aligned}
$$

and the solutions $v(x, t)$ and $w(x, t)$ are the same in the case 1 .

For $a>0, b<0$. Similarly for $a<0, b>0$. Substituting (18) along with (10) in Eq. (13) and setting all the coefficients of powers $f(\zeta)$ to zero in the resultant expression. With the aid of Mathematica, we find the special solutions of the Eq. (13) as follows

\section{Case 1:}

$$
\begin{aligned}
& c_{0}=\mp \frac{\delta \sqrt{\left(1-\beta^{2}\right) k_{1}^{2}+2 k_{1} \omega_{1}+\omega_{1}^{2}}}{\sqrt{2\left(\beta^{2}-1\right)}}, \\
& c_{1}= \pm \frac{\sqrt{2\left(\left(1-\beta^{2}\right) k_{1}^{2}+2 k_{1} \omega_{1}+\omega_{1}^{2}\right)}}{\sqrt{\beta^{2}-1}}, \\
& k_{2}=-\frac{\delta k_{1}}{\sqrt{2}} .
\end{aligned}
$$

Using solution (11) of Eq.(10). Then the exact solution to Eq.(13) can be written as 


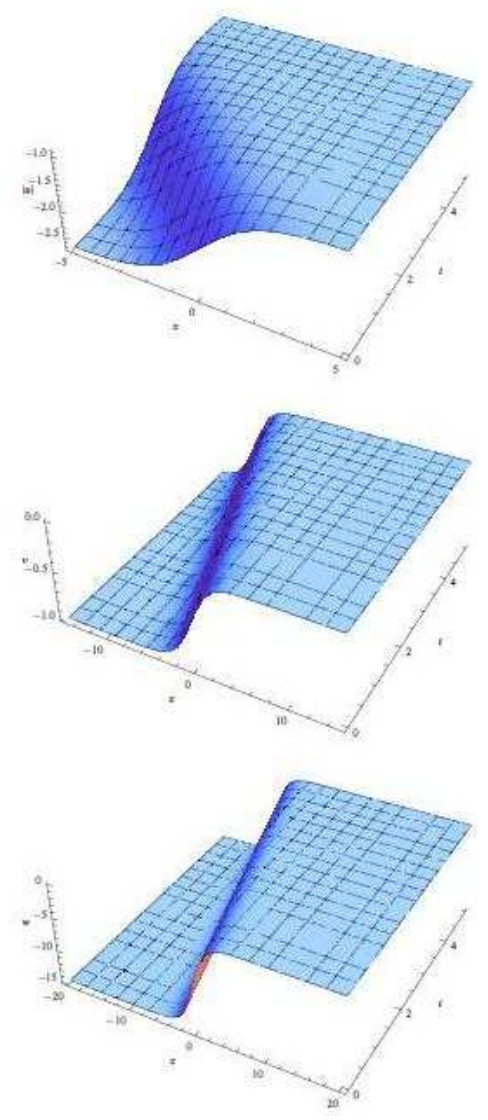

Fig. 2: The solutions of Eq.(13) when $a<0, b>0$.

$$
\begin{aligned}
u(x, t)= & \frac{\sqrt{\left(1-\beta^{2}\right) k_{1}^{2}+2 k_{1} \omega_{1}+\omega_{1}^{2}}}{\sqrt{\left(\beta^{2}-1\right)}} e^{i\left(-\frac{\delta}{\sqrt{2}}\left(k_{1} x+\left(\omega_{1}+2 k_{1}\right) t\right)+\phi_{0}\right)} \\
& \left(\frac{\delta \sqrt{2} \exp \left(\delta\left(\omega_{1} t+k_{1} x+\xi_{0}\right)\right)}{1-b \exp \left(a\left(\omega_{1} t+k_{1} x+\xi_{0}\right)\right)}-\frac{\delta}{\sqrt{2}}\right), \\
v(x, t)= & \mp \frac{\omega_{1}}{(1+\beta)\left(\omega_{1}+k_{1}(1+\beta)\right)}\left(\frac{\sqrt{\left(1-\beta^{2}\right) k_{1}^{2}+2 k_{1} \omega_{1}+\omega_{1}^{2}}}{\sqrt{\left(\beta^{2}-1\right)}}\right)^{2} \\
& \left(\left(\frac{a b \sqrt{2} \exp \left(\delta\left(\omega_{1} t+k_{1} x+\xi_{0}\right)\right)}{1-b \exp \left(a\left(\omega_{1} t+k_{1} x+\xi_{0}\right)\right)}-\frac{\delta}{\sqrt{2}}\right)\right)^{2}, \\
w(x, t)= & \mp \frac{\omega_{1}}{(1+\beta)\left(\omega_{1}+k_{1}(1+\beta)\right)}\left(\frac{\sqrt{\left(1-\beta^{2}\right) k_{1}^{2}+2 k_{1} \omega_{1}+\omega_{1}^{2}}}{\left.\sqrt{\left(\beta^{2}-1\right.}\right)}\right)^{2} \\
& \left(\left(\frac{\delta \sqrt{2} \exp \left(\delta\left(\omega_{1} t+k_{1} x+\xi_{0}\right)\right)}{1-b \exp \left(a\left(\omega_{1} t+k_{1} x+\xi_{0}\right)\right)}-\frac{\delta}{\sqrt{2}}\right)\right)^{2},
\end{aligned}
$$

when $\delta>0$.Similarly for $\delta<0$.

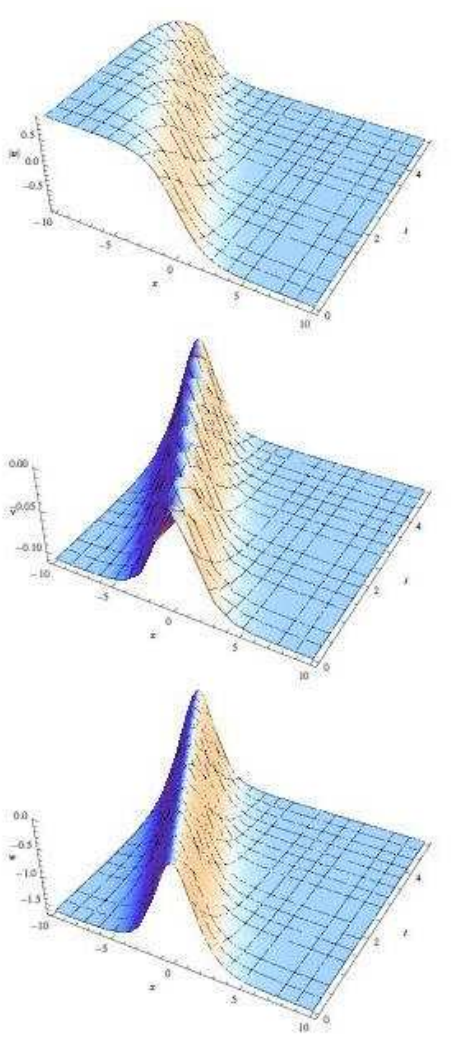

Fig. 3: The solutions of Eq.(13) when $\delta>0$.

\section{Case 2:}

$$
\begin{aligned}
& c_{0}=\mp \frac{\delta \sqrt{\left(1-\beta^{2}\right) k_{1}^{2}+2 k_{1} \omega_{1}+\omega_{1}^{2}}}{\sqrt{2\left(\beta^{2}-1\right)}}, \\
& c_{1}= \pm \frac{\sqrt{2\left(\left(1-\beta^{2}\right) k_{1}^{2}+2 k_{1} \omega_{1}+\omega_{1}^{2}\right)}}{\sqrt{\beta^{2}-1}}, \\
& k_{2}=\frac{\delta k_{1}}{\sqrt{2}} .
\end{aligned}
$$

Using solution (11) of Eq.(10). Then the exact solution to Eq.(13) can be written as

$$
\begin{aligned}
u(x, t)= & \frac{\sqrt{\left(1-\beta^{2}\right) k_{1}^{2}+2 k_{1} \omega_{1}+\omega_{1}^{2}}}{\left.\sqrt{\left(\beta^{2}-1\right.}\right)} e^{i\left(\frac{\delta}{\sqrt{2}}\left(k_{1} x+\left(\omega_{1}+2 k_{1}\right) t\right)+\phi_{0}\right)} \\
& \left(\frac{a b \sqrt{2} \exp \left(a\left(\omega_{1} t+k_{1} x+\xi_{0}\right)\right)}{1-b \exp \left(a\left(\omega_{1} t+k_{1} x+\xi_{0}\right)\right)}-\frac{\delta}{\sqrt{2}}\right)
\end{aligned}
$$

and the solutions $v(x, t)$ and $w(x, t)$ are the same in the case 1. Similarly for $\delta<0$. 


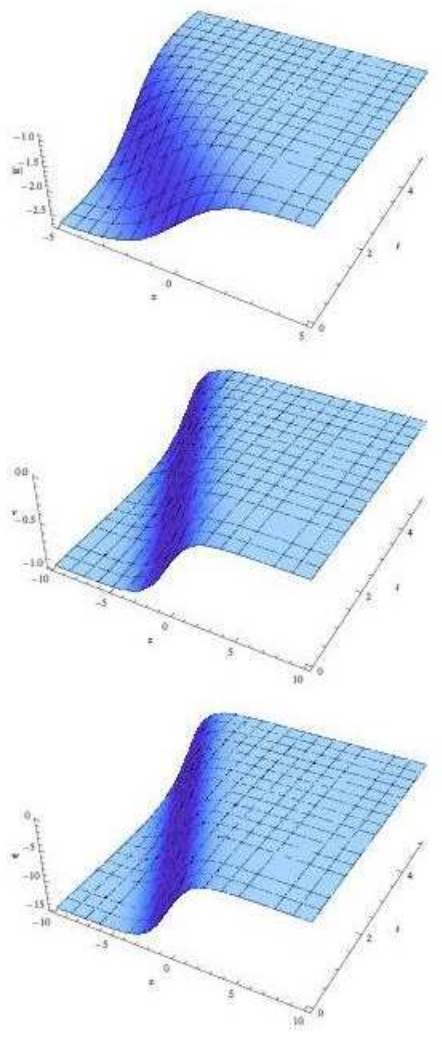

Fig. 4: The solutions of Eq.(13) when $\delta<0$.

\section{Conclusion}

In this paper, we have applied the method of the simplest equation for obtaining exact traveling wave solution of the coupled nonlinear Schrödinger type equation. We have used the equations of Bernoulli and Riccati as the simplest equations. Furthermore, the proposed method is readily computerizable by using the symbolic software. Using the proposed method, we have shown that the traveling wave solutions of a coupled nonlinear Schrödinger type equation depend on the explicit solvability of a simple system of ordinary differential equations. Finally, we point out that this method can be applied to a large class of either integrable or non-integrable nonlinear coupled systems.

\section{References}

[1] Gardner CS, Greene JM, Kruskal MD, Miura RR, Phys Rev Lett., 19, 1095-7 (1967).

[2] Ablowitz MJ, Kaup DJ, Newell AC, Phys Rev Lett., 31, 125-7 (1973).

[3] Ablowitz MJ, Kaup DJ, Newell AC, Segur H, Stud Appl Math., 53, 249-315 (1974).

[4] Hirota R, Phys Rev Lett., 27, 1192-4 (1971).

[5] W. X. Ma, Phys. Lett., A 180, 221-224 (1993).
[6] W. Malfliet, Amer. J. Phys., 60, 650-654 (1992).

[7] W. X. Ma, T.W. Huang, Y. Zhang, Phys. Scr., 82, (2010).

[8] M.R. Miura, Backlund Transformation, Springer-Verlag, Berlin, (1978).

[9] N. A. Kudryashov, Chaos Soliton Fract., 24, 1217-1231 (2005).

[10] N. A. Kudryashov, Phys. Lett., A 342, 99-106 (2005).

[11] N. K. Vitanov, Z. I. Dimitrova, H. Kantz, Appl. Math. Comput., 216, 2587-2595 (2010).

[12] Kudryashov NA, Loguinova NB, Commun. Nonlinear Sci. Numer. Simulat., 14, 3507-29 (2009).

[13] W. X. Ma, Commun. Nonlinear Sci. Numer. Simul., 16, 2663-2666 (2011).

[14] Ma YL, Geng XG, Chaos Soliton Fract, 42, 2949-53 (2009).

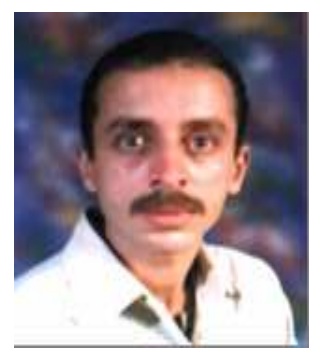

Mohammed K. Elboree obtained the $\mathrm{PhD}$ degree in applied mathematics in "The theory of Soliton", Mathematics Department, Faculty of Science, South Valley University, Qena, Egypt. He has a number of papers published in many of scientific journals. 